\title{
SENTIMENTOS E REAÇÕES DE PAIS DE CRIANÇAS EPILÉPTICAS
}

\author{
ELISABETE A. PEDROSO DE SOUZA*, CLAÚDIA RUBIN NISTA****, \\ ANNA ELISA SCOTONI***, MARILISA M. GUERREIRO**
}

\begin{abstract}
RESUMO - Objetivos: Identificar as crenças e os sentimentos dos pais frente à epilepsia e relacioná-los com os comportamentos de seus filhos. Avaliar a eficácia dos grupos de pais na diminuição da ansiedade, esclarecimento sobre a doença e comportamentos. Método: Foram aplicados 18 protocolos que avaliaram sentimentos, crenças e comportamento dos pais e filhos, respondidos antes e depois das sessões de grupos de apoio. Resultados: Diante do diagnóstico foram observados mágoa $(94,4 \%)$, medo $(72,2 \%)$, susto $(27,8 \%)$, tristeza $(33,3 \%)$ e rejeição $(38,9 \%)$. Estes sentimentos foram associados a superproteção $(83,3 \%)$ e falta de limites $(38,9 \%)$. Segurança foi associada a percepção do controle de crise. Depois do grupo, 94,4\% dos pais relatam menos ansiedade e 77,8\% associam muitas das dificuldades a falta de informação e a presença de crenças irracionais. Conclusão: Grupos de apoio desmistificam crenças, ajudam na identificação das relações parentais e previnem dificuldades comportamentais.
\end{abstract}

PALAVRAS-CHAVE: epilepsia infantil, grupo de pais, comportamento.

\section{Feelings and behaviors of parents of children with epilepsy}

ABSTRACT - Purpose: To assess the efficacy of support groups in identifying parents feelings and behaviors facing the diagnosis of epilepsy in their children. Methods: Protocols were applied to 18 parents before and after the sessions. Each protocol consisted of questions concerning feelings and beliefs toward epilepsy as well as children-parent interactions. Results: The following feelings were observed: disappointment $(94.4 \%)$, fear $(72.2 \%)$, frightening $(27.8 \%)$, sadness $(33.3 \%)$, anxiety $(27.8 \%)$ and rejection $(38.9 \%)$. These feelings were associated with overprotection (83.3\%) and a lack of limits (38.9\%). Parents reported feeling of safety after seizure control and $77.8 \%$ associate major of difficulties to the lack of information and the inadequated beliefs involved. After support sessions, $94.4 \%$ of the parents reported less anxiety. Conclusion: Support groups dispel misconception, clarify child parent relationships and prevent behavioral difficulties.

KEY WORDS: infantile epilepsy, support groups, behavior.

A epilepsia é considerada como uma experiência frustante para a família ${ }^{5}$. Seu diagnóstico gera nos familiares um desajuste emocional, mobilizando-os no sentido de se adaptarem a uma doença crônica. $\mathrm{O}$ ajustamento familiar, por sua vez, tem profundo impacto em como as crianças reagem. Crianças e adolescentes epilépticos reconhecem que têm uma condição que os diferencia de outras crianças ${ }^{4}$. Pais percebem seus filhos como imaturos e com várias dificuldades em habilidades psicológicas diversas. Eles falam de sentimentos de culpa, do medo ${ }^{10}$ gerados por crenças irracionais e desinformação ${ }^{10,16}$.

Os sentimentos de medo, ansiedade, raiva, mágoa, culpa e as reações de superproteção, permissividade, superindulgência dos pais - expressas nas excessivas restrições às atividades infantis

Departamento de Neurologia da Faculdade de Ciências Médicas (FCM) da Universidade Estadual de Campinas (UNICAMP): *Psicóloga, Professor Assistente Doutor; **Neurologista Infantil, Professor Assistente Doutor; ***Neurologista Infantil; ****Psicóloga estagiária. Aceite: 3-outubro-1997.

Dra. Elisabete Abib Pedroso de Souza - Departamento de Neurologia/FCM/UNICAMP - Caixa Postal 6111 13083-970 Campinas SP - Brasil. 
(como por exemplo: andar de bicicleta, jogar bola, brincar na casa de amigos) e nas próprias dificuldades, que sentem em impor limites disciplinares ou mesmo estabelecer punições adequadas, acabam prejudicando o desenvolvimento emocional e social de seus filhos epilépticos ${ }^{12}$. Contingentes a estas atitudes parentais é comum nestes a presença de comportamentos como insegurança, imaturidade, dependência, falta de autonomia e baixa auto estima, base das alterações psicológicas frequentes na epilepsia ${ }^{11,13,14}$. Problemas de comportamento em crianças com epilepsia são relacionados com uma variedade de fatores sociais envolvidos no processo de ajustamento, em particular, variáveis envolvendo a relação pais e filhos ${ }^{11}$.

O objetivo deste estudo foi identificar sentimentos e reações dos pais de crianças e adolescentes portadores de epilepsia e investigar, após intervenção, a mudança na percepção dos pais em relação aos seus próprios comportamentos.

\section{MÉTODO}

Foram selecionados 18 pais (16 mães e 2 pais) dentre os que participavam pela primeira vez de grupos de apoio para responderem ao protocolo relativo às suas crenças, sentimentos e reações diante da doença.

Este instrumento foi elaborado por psicóloga com 20 anos de experiência clínica hospitalar e foi baseado no relato de pais de crianças e adolescentes epilépticos sobre suas vivências com a doença (Quadro 1). Este protocolo consta de 2 partes: a primeira parte, com 15 questões, foi apresentada no início do grupo (pré-teste), e a segunda parte, com 5 questões, que foram respondidas após a intervenção (pós-teste). Tal intervenção envolveu a apresentação do vídeo educativo "Orientando os pais"15, seguido de discussão.

O vídeo traz informações médicas sobre a doença, descreve e demonstra como proceder durante as crises, elimina informações errôneas relacionadas com a epilepsia, enfatiza a importância do uso de drogas antiepilépticas e, além disso, trabalha aspectos interacionais criança/adolescente e pais na prevenção de alterações comportamentais.

As informações transmitidas no áudio-visual são discutidas em seguida e envolvem temas relacionados às dificuldades que pais sentem ao lidar com o filho epiléptico focalizando principalmente a relação pais e filhos, visando o ajustamento psicológico.

Quando a discussão focaliza temas da área médica, há orientação para que tais questões sejam escritas e os pais são estimulados a questionar o médico na consulta que segue a atividade grupal.

Grupos de apoio são formados voluntariamente por pais de crianças e adolescentes que já apresentam o diagnóstico médico de epilepsia.

Podem participar o casal ou qualquer um dos pais que estiver acompanhando a criança no dia da consulta. São grupos abertos e pais que já participaram poderão retornar para novas informações e esclarecimentos. Há pais que solicitam acompanhamento individual. Estes são acolhidos no serviço de Psicologia. Estes grupos acontecem uma vez por semana, no horário do Ambulatório de Epilepsia Infantil. São supervisionados por psicóloga e têm duração de uma hora e meia aproximadamente.

$\mathrm{Na}$ formação dos grupos, tem-se o cuidado de manter características uniformes, como por exemplo epilepsia de difícil controle ou epilepsia benigna da infância. Este estudo foi realizado com pais de crianças com epilepsia benigna da infância.

A amostra composta por 18 sujeitos foi suficiente para estimar a proporção de pais sensibilizados num intervalo de 0,15 de variação e $\alpha$ nível de significância igual a 5\%.

\section{RESULTADOS}

Frente ao diagnóstico foram relatados sentimentos de medo $(72,2 \%)$, susto $(27,8 \%)$, tristeza $(33,3 \%)$ e ansiedade $(27,8 \%)$.

Surgiram dificuldades em aceitar a doença expressando rejeição $(38,9 \%)$, sentimentos de mágoa $(94,4 \%)$ e culpa $(11,11 \%)$. 
Quadro 1. Protocolo pré-teste e pós-teste.

\section{PRÉ-TESTE}

1) O que você sentiu quando o médico disse que seu filho era epiléptico?
( ) desapontamento
( ) ansiedade
() raiva
( ) culpa
( ) confusão
() susto
() vergonha
( ) rejeição
( ) medo
( ) tristeza
( ) mágoa

2) Você percebeu alteração no comportamento do seu filho após a primeira crise?
( ) $\mathrm{Sim}$
( ) Não

3) Conta para as pessoas que seu filho tem crises?
( ) $\mathrm{Sim}$
( ) Não

4) Fica observando seu filho na maior parte do tempo?
( ) $\mathrm{Sim}$
( ) Não

5) Fica preocupado com a possibilidade da criança vir a ter uma crise inesperadamente?
( ) $\mathrm{Sim}$
( ) Não

6) Acha que seu filho é diferente de outras crianças?
( ) $\mathrm{Sim}$
( ) Não

7) Acha que falar "não" para ele pode gerar uma crise?
( ) $\mathrm{Sim}$
( ) Não

8) Costuma fazer tudo o que seu filho quer?
( ) $\mathrm{Sim}$
( ) Não

9) Acha que a epilepsia pode levar a dificuldades na escola?
( ) $\mathrm{Sim}$
( ) Não

10) Tem medo que seu filho fique com uma doença mental?
( ) $\mathrm{Sim}$
( ) Não

11) Você fica apreensivo com a possibilidade do seu filho vir a ter crises?
( ) $\operatorname{Sim}$
( ) Não

12) Protege seu filho por ter receio de que venha a ter crises?
( ) $\mathrm{Sim}$
( ) Não

13) A epilepsia é contagiosa?
( ) $\mathrm{Sim}$
( ) Não

14) Quando as crises estão controladas você fica mais tranqüila?
( ) $\mathrm{Sim}$
( ) Não

15) Você percebe que mudou o jeito de lidar com seu filho depois da primeira crise?
( ) $\operatorname{Sim}$
( ) Não

\section{PÓS-TESTE}

- Você recebeu várias informações sobre a doença.

1) Estas informações modificaram algumas crenças sobre epilepsia?

2) Esta discussão permitiu você perceber o que sente em relação a seu filho?

3) Você conseguiu perceber como costuma reagir ao comportamento dele?

4) Você acredita que estas discussões ajudam a diminuir medo e o estresse relacionados à doença?

5) Você acredita que é capaz de mudar a maneira de ver e se comportar com relação a seu filho? 
Nove pais $(50 \%)$ relatam que percebem as crises mais controladas e sentem-se mais seguros e tranquilos. Duas mães continuaram a se sentir apreensivas quanto à possibilidade de as crises voltarem apesar de seus filhos não apresentarem novas crises. Sete mães $(38,9 \%)$ cujos filhos não apresentavam crises controladas apresentaram mais respostas de medo, insegurança, preocupação diante de diferentes situações relacionadas a epilepsia, e reagiam com proteção e observação exageradas.

Os sentimentos de medo foram relacionados às interrelações sociais, aceitação na escola, desempenho acadêmico, independência e falta de informação sobre a doença.

Nos diferentes momentos em que tais sentimentos estavam presentes, geraram nos pais comportamentos de esconder a doença do filho $(38,9 \%)$, proteção exagerada $(83,3 \%)$ e permissividade $(38,9 \%)$.

Quinze pais $(83,3 \%)$ responderam pelo menos a uma das crenças pesquisadas nas questões 7 , 8,10 e 13 .

Os pais relatam que seus filhos depois que começaram a ter crises e tomar remédio apresentaram mais comportamentos negativos. Tornaram-se mais irritados $(38,9 \%)$, nervosos $(33,3 \%)$, impacientes $(27,8 \%)$ e agitados $(27,8 \%)$ (Obs: alguns pais associaram mais de um comportamento).

Aproximadamente $78 \%$ dos pais relatam que mudaram a maneira de lidar com os filhos após a primeira crise.

O pós-teste revelou que $94,4 \%$ dos pais relatam maior conhecimento sobre epilepsia, menos estresse relacionado à doença e acreditam ser capazes de serem mais adequados ao lidar com seus filhos e 77,8\% conseguiram perceber os próprios sentimentos e comportamentos em relação à epilepsia.

\section{DISCUSSÃO}

Variáveis médicas tais como história das crises podem ser fortes preditores do controle da epilepsia e condições de saúde geral ${ }^{12}$. Este estudo investigou a hipótese de que fatores sócio-culturais que incluem preconceitos, mitos relacionados à doença são associados a medos, ansiedade e atitudes paternas negativas causando problemas de comportamento nos filhos. O diagnóstico de uma doença crônica pode representar a perda de uma criança perfeita. Reações emocionais de pais diante deste fato podem incluir intensos sentimentos de medo, raiva, depressão que refletem negativamente no desempenho familiar ${ }^{17}$.

A maioria dos pais ao responder o questionário mostrou sentimentos de mágoa $(94,4 \%)$, de medo $(72,2 \%)$ e dificuldades em aceitar a doença quando verbalizam que escondem a doença do filho $(38,9 \%)$ ou explicitam a rejeição como reação ao diagnóstico $(38,9 \%)$. Tais atitudes parecem contingentes a crenças irracionais e desinformação, conforme já identificaram Souza e Guerreiro ${ }^{16}$.

Neste estudo, alguns conceitos relacionados à epilepsia são investigados, como a idéia de que a doença possa causar dificuldades na escola ou doença mental ou possa ser contagiosa e a crença de que frustações possam gerar crises, como, por exemplo, dizer "não" aos desejos dos filhos. As respostas mostraram que $83,3 \%$ dos pais tinham pelo menos uma destas crenças.

Quando falta informação sobre a doença, algumas idéias inadequadas perpetuadas culturalmente são reforçadas e acabam, em grande parte, sendo responsáveis pelos sentimentos de ansiedade e mágoa relatados pelos pais. Medo e desinformação sobre as consequências das crises podem significativamente influenciar no ajustamento comportamental e psicossocial desde que altera a maneira de lidar com o epiléptico 6 . Mitchell e col..$^{12}$ comentam que medos inapropriados e exagerados e atitudes excessivamente protetoras são mais comuns entre pais menos informados.

Este estudo mostra comportamentos de superproteção, permissividade e observação exagerados associados a sentimentos de insegurança em relação ao controle das crises.

Numa população de 18 pais, 9 sentem-se inseguros e 2 apreensivos após o controle das crises e 7 são inseguros diante da incontrolabilidade das crises. 
Hoare e Kerley ${ }^{8}$ identificaram forte associação entre a severidade da epilepsia e ansiedade materna. Por outro lado, Mitchell e col. ${ }^{12}$ observaram que um bom controle das crises não era garantia de tranquilidade. Havia relatos de medo de que as crises retornassem e relato de incerteza quanto a habilidade de mães em lidarem com a criança epiléptica. Os pais, sentindo-se confusos quanto aos limites que podiam impor a seus filhos, acreditavam que tensão e frustração desencadeavam crises e tratavam seus filhos de forma diferente.

Austin e Fraser ${ }^{2}$ e Henriksen ${ }^{9}$ reconhecem que alterações na dinâmica familiar são frequentes e condicionam na criança epiléptica comportamentos de dependência, insegurança, medo e adoção do papel de doentes. Instala-se muitas vezes um círculo vicioso entre o comportamento dos pais e criança que mantém a inadequação. As respostas à questão 2 mostram que mães percebem seus filhos como irritados, imaturos, agitados e agressivos após o início das crises e estes dados coincidem com os achados de Guerreiro e col. ${ }^{7}$. O protocolo de Qualidade de Vida aplicado identificou mudanças no comportamento significativas após o início da doença e uso de medicação.

Outros investigadores na área de epilepsia também demonstraram o significante papel que as interações mãe-criança exercem na determinação de alterações de comportamento ${ }^{1,13,14}$.

Variáveis da doença (tipo e frequência) relacionados a problemas de ajustamento social em crianças têm se mostrado secundários a processos de interações familiares nos quais a doença se manifesta, encontrando-se uma relação positiva entre atitudes de mães voltadas para a autonomia e confiança nas crianças e controle de comportamentos ${ }^{11}$. Quando pais têm a possibilidade de ser orientados sobre a doença, uso de drogas, aspectos comportamentais e sociais, sentimentos e atitudes dos pais, consequentemente há um melhor ajustamento de seus filhos ${ }^{10}$. A atenção dirigida só para o controle de crises na avaliação clínica não atende às necessidades dos pais e não controla a possibilidade de aparecimento de problemas que afetam a qualidade de vida de crianças com epilepsia ${ }^{3}$. A possibilidade de pais falarem de suas dificuldades ao lidar com a criança epiléptica alivia o estresse. Austin e Fraser ${ }^{2}$ apontam que a educação é necessária e reduz o estresse pois geralmente os medos são irracionais.

O vídeo apresentado, através de informações médicas corretas sobre o que é epilepsia, como se conduzir durante as crises, importância das drogas prevenindo o uso de procedimentos alternativos, modifica preconceitos e idéias erradas relacionadas à epilepsia. São informações muitas vezes novas para os pais, que são reforçadas nas discussões que se seguem através dos exemplos do dia-a-dia que eles próprios trazem. Assim, quando pais dizem que acreditam que a epilepsia traz dificuldades mentais, muito provavelmente têm baixas expectativas com relação a seus filhos e os solicitam pouco. Deste modo estas crianças se tornam inseguras, dependentes, medrosas e desenvolvem uma baixa auto estima.

Durante a intervenção, os pais são ensinados a perceber a relação entre suas crenças, sentimentos e reações, que são responsáveis pelos comportamentos infantis.

A questão 5 do pós-teste identifica $74,4 \%$ dos pais como mais capacitados a agir com seus filhos após serem informados sobre as condições que regem o comportamento. Existe uma predisposição dos pais a tomarem atitudes mais adequadas para modificarem seu ambiente e conduzirem a um melhor ajustamento.

Este estudo mostra a importância de fatores sócio-culturais e comportamentais no funcionamento da criança com epilepsia. Especula-se que programas educativos possam ser aplicados a outras crianças portadoras de doenças crônicas. Sugerem-se estudos longitudinais com aplicação do questionário num maior número de sujeitos e sessões de grupos para controlar o efeito da pessoa do terapêuta como variável interveniente e garantir a validade do instrumento para identificação de variáveis familiares e eficácia da intervenção em grupos de apoio. Ainda, sugerem-se pesquisas em que se deseja especificar a análise comportamental proposta, o uso de instrumentos para identificação das fontes de estresse familiar, a identificação de mitos, protocolos para avaliar o impacto da doença, o estilo de funcionamento familiar e identificação de comportamentos infantis. 
Avaliar o impacto de fatores psicossociais e comportamentais de risco para a conduta terapêutica permite estabelecer metas de controle com consequente melhora na qualidade de vida da criança epiléptica.

\section{REFERÊNCIAS}

1. Austin JK. Childhood epilepsy: child adaptation and family resources. J Child Adolesc Psychiatry Ment Health Nurs 1988; $1: 18-24$.

2. Austin JK, Fraser RT. Childrens with epilepsy: their families and later vocational adjustment. Issues in epilepsy and quality of life. Maryland: Epilepsy Foundation of America, 1993:1-8.

3. Austin JK, Smith MS, Risinger MW, Michelis AM. Childhood epilepsy and asthma: comparison of quality of life. Epilepsia 1994;25:608-615.

4. Devinsky O, Penry JK. Quality of life in epilepsy: the clinicare's view. Epilepsia 1993;34:54-87.

5. Ford CA, Gibson P, Dreifuss FE. Psychosocial considerations in childhood epilepsy. In Dreifuss (ed). Pediatric epiteptology: classification and management of seizures in the child. Massachussets: John Wright \& Sons, 1984:277-295.

6. Goldstein J, Veidenberg M, Peterson R. Fear of seizures and behavioral functioning in adults with epilepsy. J Epilepsy 1990; 3:10-16.

7. Guerreiro MM, Silva EA, Scotoni AE, Souza EAP. Qualidade de vida em epilepsia na infância. JLBE 1994;7:21-26.

8. Hoare P, Kerley S. Psychosocial adjustment of children with chronic epilepsy and their families. Dev Med Child Neurol 1991; 33:201-215.

9. Henriksen O. Special problems of children with epilepsy. Epilepsia 1988;9 (Suppl.3), S6-S9.

10. Lewis MA, Hatton CL, Salas I, Lecite B, Chiofalo N. Impact of the children's epilepsy program on parents. Epilepsia 1991;32:365-374.

11. Lothman DJ, Pianta RC. Role of child mother interacion in predicting competence of children with epilepsy. Epilepsia 1993;34:658-669.

12. Mitchell WG, Scheier LM, Baker SA. Psychosocial, behavioral and medical outcomes in children with epilepsy: a development risk factor model using longitudinal data. Pediatrics 1994;94:471-477.

13. Pianta RC, Lothman DL. Risk predicting behavior problems in children with epilepsy: child factors, disease factors, family stress and child mother interaction. Child Dev 1994;65:1415-1428.

14. Ritchie K. Interaction in the families of epileptic children. J Child Psychol Psychiatry 1981;22:65-71.

15. Souza EAP. Avaliação de vídeo educativo sobre epilepsia na infância. JLBE 1994;7:103.

16. Souza EAP, Guerreiro MM. Qualidade de vida e epilepsia. In Guerreiro CAM, Guerreiro MM (eds). Epilepsia. São Paulo: Lemos Editorial, 1993:83-90.

17. Thompson PJ, Upton D. Quality of life in family members of persons with epilepsy. In Trimble MR, Dodson E (eds). Epilepsy and quality of life. New York: Raven Press, 1994;2:19-30. 